\title{
Accelerometer-Assisted 802.11 Rate Adaptation on Mass Rapid Transit System
}

\author{
Yu-Jen Lai, Wei-Hao Kuo, Wan-Ting Chiu, Shao-Ting Chang, Hung-Yu Wei* \\ Department of Electrical Engineering, National Taiwan University \\ Taipei, Taiwan \\ *hywei@cc.ee.ntu.edu.tw
}

\begin{abstract}
In-station Wi-Fi AP deployment provides opportunistic WiFi access in underground Mass Rapid Transit (MRT) system. But such vehicular network faces the obstacle of limited connection time from the MS on the train to the BS at the station. Therefore, maximizing the throughput during the tens of second intervals becomes crucial to overcome such hindrance. To achieve the goal, we propose AccelerometerAssisted Rate Adaptation (AARA) to divide the motion of the train into four phases; each adopts a specific rate adaptation mechanism. The experiments show that the average throughput of AARA outperforms that of the conventional scheme.
\end{abstract}

\section{Categories and Subject Descriptors}

C.2.1 [Computer-Communication Networks]: Network Architecture and Design-Wireless communication

\section{General Terms}

Experimentation, Design, Performance

\section{INTRODUCTION}

The demands for the Internet access in MRT systems are increasing over time. Consider the scenario of Fig. 1, BS is located at stations to provide Wi-Fi service to MS on trains. However, due to the limited transmission range, the signal quality varies highly when the train arrives at or departs from the station; therefore, the MS should quickly adjust its transmission rate to gain higher throughput. Although numerous rate adaptation mechanisms are presented, e.g. [1, $2,3]$, few of them focus on the rate adaptation in vehicular environments, especially for MRT systems.

Accelerometers are widely equipped for kinds of portable devices such as iPhone. We are able to estimate the motion of trains according to the measured acceleration. In our previous work, we designed an accelerometer-assisted power saving mechanism to trigger MS switching to sleep mode during inter-station periods [4], but not focusing on the transmission performance. In this work, we propose an accelerometer-assisted rate adaptation mechanism (AARA) to boost the throughput when MS is in the coverage of BS during the near-station and in-station phases.

Copyright is held by the author/owner(s).

SIGCOMM'10, August 30-September 3, 2010, New Delhi, India. ACM 978-1-4503-0201-2/10/08.



Figure 1: Wi-Fi access in MRT station

\section{MECHANISM OF AARA}

In this section, we will introduce the two parts of AARA's mechanism: phase estimation and rate adaptation scheme.

\subsection{Phase Estimation by Accelerometer}

Different from our previous work [4] using the vibrative acceleration to estimate two phases, the in-station and interstation phases, AARA estimates the phases more delicately to adjust the bit rates. On the basis of Moving Acceleration, AARA can estimate the four phases of the train's motion: enter phase, stop phase, start phase, leave phase, and each holds different channel characteristics. Besides, AARA also works in high capacity MRT systems in which the train's vibration alleviates much, and thus it is hard to estimate the phases by vibrative acceleration in such system.

As direction of users' accelerometers is unpredictable, we consider all the 3 -axis accelerations at the same time. We define the Moving Acceleration as:

$$
\text { M-Acc }=\mid \text { LP-Filter }(\hat{A})-\vec{G}-\vec{D}_{o f f}|-| \vec{D}_{\text {calib }} \mid,
$$

where $\hat{A}$ is the original measurement of the 3 -axis accelerations, $\vec{G}$ is the gravitation, $\vec{D}_{\text {off }}$ is the bias offset of the accelerometer, and $\vec{D}_{\text {calib }}$ is the offset due to the user's slight rotation during the time. Fig. 2 shows the pattern of M-Acc during a train passing stations: In stop phase, M-Acc is stable. In start phase, the M-Acc starts to increase and reaches the maximum as changing to leave phase. In leave phase, M-Acc is stable most of the time, only with a few sharp fluctuations. In enter phase, M-Acc gradually decreases in the negative region and then increases. We apply the exponential moving average with $\alpha=0.075$ as a LP-Filter to eliminate the abrupt vibration. The result shows M-Acc has the high correlation with the speed changes of the train. 




Figure 2: The raw data of $\mathrm{x}, \mathrm{y}, \mathrm{z}$ axis acceleration, M-Acc and the estimated phase. The dotted lines show the actual movement phase.



Figure 3: The procedure of AARA

\subsection{Rate Adaptation Scheme}

We implement AARA by modifying SampleRate bit rate selection algorithm [1], the default rate control algorithm of MadWifi. The SampleRate algorithm maintains a table of the average successful transmission time of each rate scheme, and then chooses the bit rate scheme with the shortest expected transmission time to transmit. When the train approaches a station, there is no history record yet. In enter phase, SampleRate chooses the highest rate to transmit, e.g. $54 \mathrm{Mbps}$ in 802.11g. However, the channel is poor due to the remote distance then, and the transmission success rate is low. We know that the channel quality is unstable while the train entering the station, and the MS should adopt a more robust bit rate. Thus, AARA probes from lower bit rates instead and increases the probing rate to adapt to the changing channel.

When the train stops, the wireless channel becomes stable, so the MS should quickly change to the highest rate to maximize the throughput. However, due to the successive failure of probing high rate in the previous phase, SampleRate still considers the channel quality poor, thereby using the lower rate to transmit. AARA cleans the stale record and probes from the highest rate, and once the MS reaches the optimal bit rate, the extra probing packets are reduced to remain at the optimal rate. When the train starts moving, the channel quality degrades promptly as the train speeds

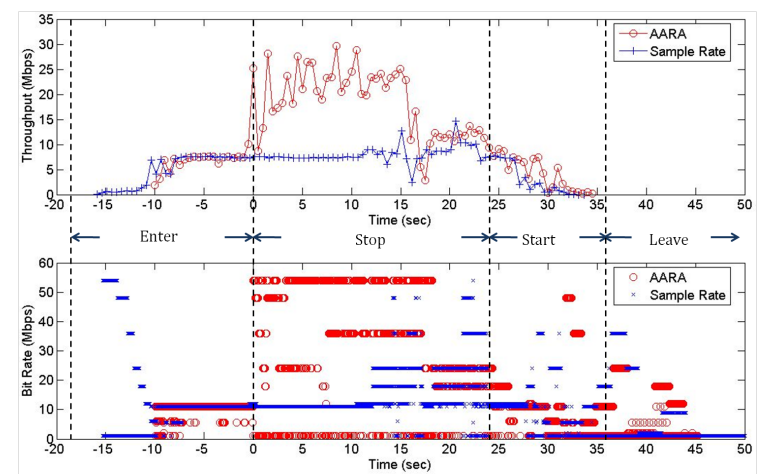

Figure 4: The throughput and the rate selection of AARA and SampleRate along the path

up, the MS should sense the unstable environment and automatically adapt to the channel again. Fig. 3 illustrates the pseudo code of AARA's procedure.

\section{EXPERIMENTAL EVALUATION}

We test the performance of AARA and SampleRate on Taipei MRT. We compare AARA with SampleRate under the same conditions. The BS is located at Xiaonanmen Station. The MS on the train moving from Ximen to CKS Memorial Hall station, and stops at Xiaonanmen in between for a while. Fig. 4 shows that SampleRate sends out many probing packets with higher rates in vain when the MS is not close enough to the BS, while AARA starts its probing from lower rates. After the train stops, the channel turns stable, and thus AARA cleans its history record and switch to higher rates timely to maximize the throughput. On the other hand, since SampleRate accumulates many successive failures of transmission at higher rates in the enter phase, it keeps using lower rates for about 21 seconds in Fig. 4 until the train is about to leave. The experiment result shows the average throughput of AARA is $12.46 \mathrm{Mbps}$ while SampleRate's 5.86 Mbps during the four phases, which indicates AARA has better adaptation to MRT.

\section{CONCLUSION}

This paper presents AARA, an adaptive rate adaptation mechanism assisted by accelerometer for MRT system. AARA uses accelerometer to estimate the four phases for better rate adaptation. Experiment results show that the throughput of AARA doubles that of SampleRate on Taipei MRT system.

\section{REFERENCES}

[1] J. C. Bicket. Bit-rate Selection in Wireless Networks. Master's thesis, MIT, 2005.

[2] S. H. Y. Wong, H. Yang, S. Lu, and V. Bharghavan. Robust Rate Adaptation for 802.11 Wireless Networks. In ACM MobiCom'06. ACM, 2006.

[3] G. Judd, X. Wang, and P. Steenkiste. Efficient Channel-aware Rate Adaptation in Dynamic Environments. In ACM MobiSys'08. ACM, 2008.

[4] S.-T. Chang, Y.-C. Huang, and H.-Y. Wei. Accelerometer-Assisted Power Efficient Wi-Fi Networking on Public Transportation System. In $A C M$ MobiCom'09 Poster. 